\title{
Impact Attenuation Capabilities of Football and Lacrosse Helmets
}

3 Katherine M. Breedlove, PhD, ATC 1

4 Evan L. Breedlove, $\mathrm{PhD}^{2}$

5 Thomas G. Bowman, PhD, ATC ${ }^{3}$

6 Eric A. Nauman, $\mathrm{PhD}^{2,4,5}$

8 1. Department of Health and Kinesiology, Purdue University, West Lafayette, IN 47907

9 2. School of Mechanical Engineering, Purdue University, West Lafayette, IN 47907

10 3. Department of Athletic Training, Lynchburg College, Lynchburg, VA 24501

11 4. Weldon School of Biomedical Engineering, Purdue University, West Lafayette, IN

1247907

13 5. Department of Basic Medical Sciences, Purdue University, West Lafayette, IN

$14 \quad 47907$

15

\section{Corresponding Author:}

Eric A. Nauman

School of Mechanical Engineering

Purdue University

20585 Purdue Mall

21 West Lafayette, IN 47907-2088

22 Tel: 7654948602

23 Fax: 7654940539

24 Email: enauman@purdue.edu

25

26 Key Words: Protective equipment, Gadd Severity Index, head impact, drop test, helmet

27 evaluation. 
ABSTRACT

31 Although the National Operating Committee on Standards for Athletic Equipment

32 (NOCSAE) standards are similar for football and lacrosse helmets, it remains unknown

33 how helmets for each sport compare on drop tests. Due to the increased concern over

34 head injury in sport and the rapid growth in lacrosse participation, it is useful to compare

35 the performance of various football and lacrosse helmets. Therefore, the goal of this

36 study was to document the impact attenuation properties of football and lacrosse

37 helmets and to identify the relative performance between helmets for the two sports.

38 Six models of football and six models of lacrosse helmets were tested using a drop

39 tower at three prescribed velocities and six locations on the helmets. A repeated

40 measures ANOVA was conducted to determine the effect of sport on Gadd Severity

41 Index (GSI) scores and linear accelerations. The interaction between location and sport

42 was significant at the low $\left(F_{2.64,89.71}=7.68, P<.001, \eta^{2}=.025\right)$, medium $\left(F_{2.85,96.85}=16.78\right.$,

$\left.43 P<.001, \eta^{2}=.085\right)$, and high $\left(F_{2.96,100.69}=16.67, P<.001, \eta^{2}=.093\right)$ velocities for $G S I$

44 scores. When comparing peak acceleration results, we found a significant interaction

45 between location and sport for the medium $\left(F_{3.40,115.616}=5.57, P=.001, \omega^{2}=.031\right)$ and high

$46 \quad\left(F_{3.46,117.50}=6.42, P<.001, \omega^{2}=.047\right)$ velocities. Features of football helmet design

47 provide superior protection compared to lacrosse helmets. Further investigation of

48 helmet design features across sports will yield insight into how design features influence

49 helmet performance during laboratory testing. 
INTRODUCTION

51 The National Operating Committee on Standards for Athletic Equipment

52 (NOCSAE) maintains standards to certify the safety of athletic helmets. Helmets that

53 meet certain impact attenuation thresholds are awarded the NOCSAE seal of approval

54 (NOCSAE, 2013a, b). These thresholds were originally developed in 1973 with the aim

55 of preventing life-threatening head injuries, such as subdural hematomas and skull

56 fractures, with considerable success (Bachynski and Goldberg, 2014; Levy et al., 2004).

57 It should be noted, however, that the standard does not specifically address

58 concussions or, of more recent interest, subconcussive impacts. Since the drafting of

59 the original standard, NOCSAE has also written standards for helmets and athletic

60 equipment used in other sports, such as lacrosse (NOCSAE, 2011, 2012).

61 These standards employ similar testing methods and criteria despite the fact that

62 the equipment and style of play are distinct between football and lacrosse. Both are

63 required to have Gadd Severity Index (GSI) scores under 300 during low velocity drops

64 and under 1200 during medium and high velocity drops (NOCSAE, 2012, 2013a). GSI

65 is generally a good indicator of severe neurotrauma risk (Goldsmith and Monson, 2005),

66 and these thresholds were set to ensure that the helmets would reliably prevent injuries

67 such as skull fracture. In other words, these thresholds are not, nor should they be,

68 sport specific nor are they meant to evaluate protection against a concussion.

69 Additionally, helmets are required not to break, crack, or suffer any structural damage

70 that results in un-restorable alterations to helmet fit during testing (NOCSAE, 2012,

71 2013a). Currently the National Collegiate Athletic Association (NCAA) and the National

72 Federation of High School Sports (NFHSS) require that helmets used in competition 
73 meet the NOCSAE standard (Hillman, 2005); therefore, manufacturers frequently cite

74 performance on the standard test as evidence of the capabilities of their helmets (Levy

75 et al., 2004). Despite the importance of helmets to overall athlete health during

76 participation in contact sports and the national requirements that helmets be certified,

77 there is little published data characterizing the performance of athletic helmets and the

78 effects of design changes.

79 Bishop et al (1984) performed an important set of experiments on football

80 helmets that evaluated the relative abilities of suspension, padded-suspension, and

81 padded helmets to mitigate impacts according to a modified NOCSAE test protocol.

82 They found that the padded helmets achieved the best results, but the results were

83 difficult to generalize because the helmets had all been in use for 6-8 years and they

84 only examined a single drop height and a single location on each helmet. More recently,

85 others compared the response of a NOCSAE headform on a Hybrid III neckform when

86 struck by a swinging, helmeted NOCSAE headform (Bartsch et al., 2012). They

87 examined five impact locations using three different impact speeds and found that

88 leather helmets performed as well or better than modern helmets in terms of reducing

89 linear, and angular accelerations. In lacrosse, the 1996-1997 season saw a substantial

90 shift in design as manufacturers dramatically changed helmet designs to adopt a lighter

91 and more streamlined look (Caswell and Deivert, 2002) that improved vision and

92 cervical mobility (Dick et al., 2007). This contemporary aesthetic attenuated impacts

93 better than the previous designs only at the right rear boss drop position, and GSI

94 scores still increased sharply upon repetitive impacts for both designs (Caswell and

95 Deivert, 2002). 
In view of the existing research on helmet performance and concussion

97 prevention, the following axioms were adopted for this research. First, all athletic

98 helmets should be able to fulfill the GSI thresholds laid out by NOCSAE, as the

99 thresholds for severe neurotrauma are not sport specific. Second, helmets should

100 maximize the degree to which they mitigate impacts while maintaining their functionality

101 for sport-specific applications. It has been suggested that impact mitigation is related to

102 a reduced risk of concussion (Rowson and Duma, 2011); therefore, it is important to

103 better understand the impact response of helmets used in contact sports. Many factors

104 influence the risk of concussion, and differences in gameplay influence the relative

105 importance of those risk factors. While current biomechanical metrics may not capture

106 all aspects of concussion risk, they are nevertheless valuable in evaluating the

107 biomechanics of head impacts. Many helmet evaluation studies focus on drawing the

108 best conclusions possible from the available metrics (Hutchinson, 2014; Post et al.,

109 2013; Rowson and Duma, 2011; Scher et al., 2008). Accordingly, this paper aims to

110 report typical values for many commonly worn helmets with regards to their

111 performance on a NOCSAE-style drop test with respect to the metrics of GSI and peak

112 linear acceleration. These metrics are relevant to football, lacrosse, or any other

113 contact sport simply due to their descriptive, though certainly not comprehensive,

114 nature. Such basic knowledge is essential to further investigations of helmet

115 performance.

116 Finally, it is observed that the sport-specific design trends in athletic helmets

117 reflect a combination of historic/aesthetic design inertia and true sport-specific

118 requirement differences (Newman, 2015). However, helmets may be viewed more 
119 generally as devices which mitigate the effect of head impacts on neural health. While

120 some design requirements differ between sports, this fundamental function is universal,

121 and the effectiveness or ineffectiveness of technologies in one lineage of sports helmets

122 is potentially relevant to other helmet types. Such an approach is valuable to engineers

123 and biomechanicians as they evaluate trends in helmet performance and new

124 technologies. It is also relevant to clinicians who are frequently faced with evaluating

125 the claims of constantly changing helmet technology in sport.

126 Therefore, this paper compares the performance of football and lacrosse helmets

127 with respect to GSI and peak linear acceleration during drop tests. While differences in

128 impact performance between helmets of various sports has long been assumed, this

129 paper investigates these discrepancies and begins to explore why variations exist.

130 Such a cross-sport analysis has not been reported to our knowledge, and similar

131 studies could be conducted to compare with other helmeted contact sports, such as

132 hockey. Through this cross-sport comparison approach, it is hoped that effective design

133 features will be identified and incorporated across sports where possible.

\section{METHODS}

\section{Helmets}

137 A total of 36 football and lacrosse helmets of various brands and models were

138 tested (Table 1). All the helmets used for this study were size large or one size fits most

139 for some lacrosse models. In addition, all helmets were new and never used for athletic

140 activity. A power analysis was conducted using a previously published study with

141 similar data (Bowman et al., 2015) which demonstrated three helmets per group were 
142 adequate to detect differences in impact attenuation ( $\alpha$ value of 0.05 and $1-\beta=0.80$ ).

143

144 Table 1. Helmets included in the study. All helmets were new and never used in

145 athletic activity.

\begin{tabular}{cccc}
\hline Sport & Brand & Model & Number \\
\hline \multirow{5}{*}{ Football } & $\begin{array}{c}\text { Riddell (Rosemont, } \\
\text { Illinois) }\end{array}$ & $\begin{array}{c}\text { Revolution } \\
\text { Speed }\end{array}$ & 3 \\
& $\begin{array}{c}\text { Schutt (Litchfield, } \\
\text { Illinois) }\end{array}$ & lon 4 & 3 \\
\cline { 2 - 4 } & & \\
\cline { 2 - 4 } & Xenith (Lowell, & X1 & 3 \\
& Massachusetts) & X2E & 3 \\
& & Epic & 3 \\
\hline \multirow{5}{*}{ Lacrosse } & Pro7 & 3 \\
& Cascade (Liverpool, & CPX & 3 \\
& New York) & CPX-R & 3 \\
& & R & 3 \\
\cline { 2 - 4 } & Warrior (Warren, & Trojan & 3 \\
& Michigan) & Venom & 3 \\
\hline
\end{tabular}

146

147

148 Instruments

149 A drop tower was constructed based on NOCSAE standards and used for all

150 drop tests (NOCSAE, 2012) (Figure 1). The tower featured an anvil with a $1 / 2$-inch

151 polymer pad against which a headform (18.415 cm circumference) fitted with a helmet

152 was impacted. The headform was attached to a carriage that runs along guide wires.

153 The headform is repositionable for six standard impact orientations (Figure 2). A triaxial

154 accelerometer (PCB Piezotronics, Inc. Depew, NY) placed at the headform center of

155 mass measured impact events. An analog filter (Cadex; Quebec, Canada) meeting the

156 80-channel, class 1000 filter with $1000 \mathrm{~Hz}$ corner frequency as required by NOCSAE

157 (NOCSAE, 2013b) preprocessed acceleration signals and also operated a time gate, 
158 which measured impact velocity just prior to impact. Filtered acceleration and velocity

159 data were sampled at $10 \mathrm{kHz}$ by a National Instuments (Austin, TX) data acquisition

160 board, which was controlled by a LabVIEW program (National Instruments). A custom

161 Matlab (Mathworks, Newton, MA) program conducted data post-processing.

162

163 Data Collection Procedures

164 We appropriately fit all helmets to a medium (circumference $18.415 \mathrm{~cm}$ )

165 headform and dropped them at the NOCSAE prescribed velocities of 3.46 (low), 4.89

166 (medium), and 5.47 (high) $\mathrm{m} / \mathrm{s}$ with 75 seconds $+/-15$ seconds between

167 drops.(NOCSAE, 2011, 2012, 2013a, b)

168 We dropped the helmets in the six orientations prescribed by NOCSAE

169 (NOCSAE, 2012, 2013a, b): Front, Right Front Boss, Top, Right Rear Boss, Rear, and

170 Side. In order to facilitate comparisons between the two sport helmets, we tested all

171 helmets at ambient temperature (range $70.7-71.6^{\circ} \mathrm{F}$ ) on all six locations at the three

172 prescribed speeds. Although we tried to follow the NOCSAE guidelines as much as

173 possible, these tests were not intended to be NOCSAE certification tests, and we did

174 not conduct any high temperature testing, randomized drops, nor the football

175 recommended drop velocity of $4.23 \mathrm{~m} / \mathrm{s}$.

176 We took several safeguards to assure measurement accuracy. First, we

177 calibrated the triaxial accelerometer using a PCB 394C06 uniaxial calibrator for each of

178 the three axes (PCB Piezotronics, Inc. Depew, NY). We completed system checks,

179 measuring velocity, peak acceleration, and GSI of the unhelmeted headform at the

180 beginning, after any change to the headform orientation, after we tested a maximum of

181 four helmets, and at the end of testing. For a system check to pass, the velocity, peak 
182 acceleration, and GSI of the tests before or after a testing segment could differ by no

183 more than $7 \%$. This was done to ensure that the configuration of the testing apparatus

184 had not significantly changed across the drops. A failed system check resulted in

185 discarding of that testing segment between the two tests and recollection of the data.

186 There was no procedural accounting for any possible location ordering effect

187 because the authors believed that all of the impacts sustained by the helmets during

188 testing were within the expected range of athletic helmets and thus drop order should

189 not affect the outcomes of this study. Velocity ordering was consistent among all

190 samples. Velocity increased from lowest to highest with a fixed time interval between

191 each impact, which is consistent with the NOCSAE standard and was put in place to

192 mitigate differences in viscoelastic recovery

193 Similar to the NOCSAE standard, a helmet failed if any GSI exceeded 1200 , if

194 the low velocity ( $3.46 \mathrm{~m} / \mathrm{s})$ GSI exceeded 300 , or if any permanent structural changes,

195 such as cracking or breaking of the suspension system, were observed (NOCSAE,

$1962012,2013 a, b)$.

197

198 Statistical Analysis

199 We used a repeated measure ANOVA to determine the effect of helmet sport on

200 GSI scores at each of the 3 prescribed velocities using IBM SPSS Statistics (version 21;

201 IBM Corporation, Armonk, NY). We used drop location as the repeated variable and set

202 the alpha level to .05 a priori. When we discovered significant interactions, we used a

203 1-way ANOVA to determine where significant differences occurred, paired samples t-

204 tests to determine GSI score differences between sports for a given location, and 
205 calculated descriptive statistics including means and 95\% confidence intervals (Cls)

206 using SPSS. In addition, we also examined the effect of helmet sport on peak

207 accelerations of the headform at all 3 velocities using the same statistical approach.

\section{RESULTS}

We collected data from 36 individual helmets at 3 velocities across 6 impact

211 locations for a total of 648 impacts. Nine lacrosse helmets failed to meet the NOCSAE

212 standard (failure could result from a GSI exceeding 1200 for any velocity drop, a GSI

213 exceeding 300 for the lowest velocity, or damage such as a crack in the helmet

214 construction). Six helmets exceeded the $1200 \mathrm{GSI}$ threshold, one helmet sustained

215 permanent structural damage, and two helmets exceeded the $1200 \mathrm{GSI}$ threshold and

216 suffered structural damage (this was responsibly disclosed to NOCSAE prior to

217 publication (Breedlove et al., 2013)). No football helmets failed to meet the standard.

219 GSI

220 A repeated measures ANOVA indicated that the interaction between location and

221 sport was significant at the low $\left(F_{2.64,89.71}=7.68, P<.001, \omega^{2}=.025\right)$, medium

$222\left(F_{2.85,96.85}=16.78, P<.001, \omega^{2}=.085\right)$, and high $\left(F_{2.96,100.69}=16.67, P<.001, \omega^{2}=.093\right)$

223 velocities (see Figure 3). Mauchly's test of sphericity was significant at each velocity,

224 indicating that data were heteroskedastic for different impact locations leading us to use

225 the Greenhouse-Geisser correction when interpreting results.

226 We used a one-way ANOVA to follow-up the significant interactions by

227 comparing the GSI scores of the football and lacrosse helmets across the 6 locations 
228 (see Figure 3). We found that the football helmets outperformed the lacrosse helmets

229 at every comparison except at the right rear boss position during the high velocity

230 testing $(P<.003)$.

231 Pairwise comparison of GSI scores within each sport and at each velocity

232 indicated significant differences between locations (Table 2). In short, the front and top

233 locations had particularly high GSI scores for the lacrosse helmets. 
235 Table 2. P-values for comparisons of the GSI scores among the different locations during low, medium, and high velocity 236 testing with football and lacrosse helmets.

\begin{tabular}{|c|c|c|c|c|c|c|}
\hline \multirow[b]{2}{*}{ Comparison } & \multicolumn{3}{|c|}{ Football } & \multicolumn{3}{|c|}{ Lacrosse } \\
\hline & $\begin{array}{l}\text { Low } \\
\text { Velocity }\end{array}$ & $\begin{array}{l}\text { Medium } \\
\text { Velocity }\end{array}$ & $\begin{array}{l}\text { High } \\
\text { Velocity }\end{array}$ & $\begin{array}{l}\text { Low } \\
\text { Velocity }\end{array}$ & $\begin{array}{l}\text { Medium } \\
\text { Velocity }\end{array}$ & $\begin{array}{l}\text { High238 } \\
\text { Velocity }\end{array}$ \\
\hline Front - Front Right Boss & .004 & .058 & .012 & .004 & $<.001$ & $<.00239$ \\
\hline Front - Side & .710 & .207 & .012 & $<.001$ & $<.001$ & $<.001$ \\
\hline Front - Right Rear Boss & .191 & .286 & .363 & $<.001$ & $<.001$ & $<.00 T^{40}$ \\
\hline Front - Rear & .873 & .422 & .044 & .293 & .001 & $<.00241$ \\
\hline Front - Top & .264 & .018 & .607 & $<.001$ & .004 & .847 \\
\hline Right Front Boss - Side & .288 & .758 & .298 & .966 & .880 & .977242 \\
\hline Right Front Boss - Right Rear Boss & .963 & .907 & .377 & .006 & $<.001$ & $<.001$ \\
\hline Right Front Boss - Rear & .266 & .720 & .499 & .336 & .568 & $.095^{243}$ \\
\hline Right Front Boss - Top & .011 & $<.001$ & .003 & $<.001$ & $<.001$ & $<.00144$ \\
\hline Side - Right Rear Boss & .003 & .595 & .049 & .003 & .065 & .014 \\
\hline Side - Rear & .531 & .265 & .574 & .422 & .849 & .471245 \\
\hline Side - Top & .004 & $<.001$ & $<.001$ & $<.001$ & $<.001$ & $<.001$ \\
\hline Right Rear Boss - Rear & .006 & .764 & .159 & .005 & .014 & $.004^{246}$ \\
\hline Right Rear Boss - Top & $<.001$ & $<.001$ & .075 & $<.001$ & $<.001$ & $<.00147$ \\
\hline Rear - Top & .030 & $<.001$ & .003 & $<.001$ & $<.001$ & $<.001$ \\
\hline
\end{tabular}




\section{Peak Accelerations}

We also found significant interactions between location and sport at the medium

$253 \quad\left(F_{3.40,115.616}=5.57, P=.001, \omega^{2}=.031\right)$ and high $\left(F_{3.46,117.50}=6.42, P<.001, \omega^{2}=.047\right)$

254 velocities (see Figure 4) for peak accelerations. Main effects for sport $\left(F_{1,34}=140.88\right.$,

$\left.255 P<.001, \omega^{2}=.518\right)$ and location $\left(F_{2.75,93.41}=4.49, P=.007, \omega^{2}=.030\right)$ were also significant

256 during the low velocity drops. Similar to the GSI analyses, Mauchly's test of sphericity

257 was significant at each velocity, leading us to use the Greenhouse-Geisser correction 258 when interpreting results.

We used a one-way ANOVA to follow-up the significant interactions by

261 comparing the peak acceleration scores of the football and lacrosse helmets across the

2626 locations (see Figure 4). We found that the football helmets produced lower peak

263 accelerations than the lacrosse helmets at every comparison except at the right rear

264 boss position during the medium $(P<.003)$ and high velocity testing $(P<.001)$. In

265 addition, we found significant differences in peak accelerations between drop locations

266 within each sport during 5 of the 15 pairwise comparisons at the low velocity $(P<.01), 9$

267 of 15 at the medium velocity $(P<.015)$, and 8 of 15 comparisons at the high velocity $268(P<.049)$.

\section{DISCUSSION}

271 Lacrosse is currently one of the fastest growing sports in North America (Hinton 272 et al., 2005) with a net gain of 174 programs at the collegiate level between 1988 and 
2732014 as compared to 67 new football programs (NCAA, 2013). High participation rates--

27432,500 athletes playing intercollegiate football and lacrosse and over 1.1 million at the

275 high school level--warrant an in-depth investigation in helmet design and efficacy. The

276 goal of this study was to evaluate helmets currently in use for both sports in order to

277 determine the relative impact mitigation that each style of helmet provides. We found a

278 dramatic difference in the GSI values and peak accelerations of lacrosse and football

279 helmets tested according to a protocol based on the NOCSAE standard as of 2014. In

280 addition to the lacrosse helmets exhibiting significantly higher values of GSI and peak

281 linear acceleration at all but one of the combinations of test location and impact velocity,

282 half the lacrosse helmets tested failed to meet the NOCSAE criterion. These findings

283 were responsibly disclosed to NOCSAE in November 2013-prior to submission of the

284 manuscript and prior to the 2014 recall of some affected models (Breedlove et al.,

285 2013). Independent testing confirmed the failing performance by the lacrosse helmets

286 when they were subjected to the full range of tests (STX).

287 Previous research on head impacts in football indicate that, overall, football

288 players sustain more head impacts than lacrosse players (Duma et al., 2005; Higgins

289 and Bowman, 2015; Koehling et al., 2015; Martini et al., 2013; O’Day et al., 2015;

290 Vollavanh et al., 2015). However, the accelerations of the head impacts that do occur in

291 both sports are comparable to the linear accelerations obtained in the low and medium

292 velocity drop tests (Duma et al., 2005; Martini et al., 2013; Vollavanh et al., 2015).

293 Since this study was not meant to evaluate the effectiveness in helmet attenuation over

294 time, we believed it was useful to compare across testing. However, the clinical

295 implications of reduced GSI scores under the NOCSAE threshold of $1200 \mathrm{GSI}$ remain 
296 unknown. It has been found that the average linear accelerations in NCAA division I

297 and division III lacrosse players were near $30 \mathrm{~g}$ for both practices and games (Higgins

298 and Bowman, 2015) while average linear accelerations were approximately $28 \mathrm{~g}$ for

299 high school (Breedlove et al., 2012) and $22.25 \mathrm{~g}$ for collegiate (Mihalik et al., 2007)

300 football players. These relatively low accelerations result in GSI values nowhere near

301 1200, yet are still associated with subconcussive neurophysiological changes and an

302 increased risk of concussion (Breedlove et al., 2012).

303 Based on the connections between drop testing results and possible concussive

304 risk (Rowson and Duma, 2011) as well as the evidence that head impact magnitude, but

305 not frequency, is similar between football and lacrosse (Duma et al., 2005; Higgins and

306 Bowman, 2015; Koehling et al., 2015; Martini et al., 2013; O'Day et al., 2015; Vollavanh

307 et al., 2015), there is justification to expect that football and lacrosse helmets need to

308 attenuate impact forces similarly. Consequently, we believe lacrosse helmet

309 modifications to reduce GSI scores are warranted. While a systematic study has not

310 been conducted to identify football helmet design features that improve impact

311 attenuation, there appear to be aspects of football helmet design that could be

312 integrated into lacrosse helmets to improve their performance. Notable differences in

313 design that may account for the performance disparity include pad material and

314 thickness as well as shell material and stiffness (Bower et al., In press; Bowman et al.,

315 2015; Martin et al., 1994).

316 We believe it is noteworthy that the front and top drop locations had particularly

317 high GSI scores for the lacrosse helmets. Previous work identified a correspondence

318 between front and top impacts and subconcussive changes in neurophysiology 
319 (Breedlove et al., 2012; Talavage et al., 2014), and additional research identified a

320 correlation between concussion severity and strains in the frontal regions of the brain

321 (Ommaya and Hirsch, 1971). Additionally, the majority of concussions were caused by

322 front or top impacts to the helmet in one study of collegiate football (Guskiewicz et al.,

323 2007), and hits to the facemask caused the second most concussions next to the side in

324 a high school lacrosse study (Lincoln et al., 2013). Thus, the front and top locations of

325 the helmet should be manufactured to mitigate impact forces in an effort to reduce

326 clinical sequelae. In addition, perhaps behavioral modification of athletes can help

327 reduce the number and magnitude of impacts to these locations.

$328 \quad$ Athletic helmets and the associated NOCSAE drop tests were designed to

329 prevent life threating injuries such as skull fracture, subdural hematomas, and others

330 (Gadd, 1966). All football and lacrosse helmets must meet the NOCSAE standards

331 which hold the same GSI threshold for football and lacrosse helmets (NOCSAE, 2013b).

332 Therefore it was disturbing that we found some lacrosse helmets failed to meet the

333 NOCSAE standard for protective lacrosse helmets (NOCSAE, 2012).

334

335 Limitations and Future Directions

336 It is important to note some limitations to the current study. While we attempted

337 to choose a wide range of helmets currently available, our sample was not

338 comprehensive. As helmet manufacturers continue to strive to make better products,

339 new helmet models should also be studied. Further, we only performed the drop testing

340 protocol on 3 helmets from each brand or style. However, NOCSAE standards require

341 statistical process control of certified helmets; therefore, we believe the variability 
342 among helmets within a particular brand should be minimal. The NOCSAE has also

343 released new standards for testing helmets with a lateral impactor to better reflect the

344 forces that have been shown to cause injury in football players (NOCSAE, 2006). It

345 would be interesting to see how football and lacrosse helmet lateral impactor test results

346 would compare.

\section{CONCLUSIONS}

While football helmets generally exhibited GSI values well below the NOCSAE

350 threshold of 1200, lacrosse helmets were consistently close to the threshold and in 8

351 cases exceeded the threshold, in violation of NOCSAE standards. Features of football

352 helmet design provide superior protection compared to lacrosse helmets. Such a cross-

353 sport analysis is novel, and further investigation of helmet design features across sports

354 will yield insight into how design features influence helmet performance during

355 laboratory testing. NOCSAE testing results are not generally published in the literature;

356 however, this research indicates that all helmets that receive NOCSAE certification are

357 not equal in their ability to attenuate head impacts. Open publication of testing results

358 provides valuable information to decision makers who select helmets for the protection

359 of athletes.

\section{ACKNOWLEDGEMENTS}

This work was supported in part by grants from the Indiana State Department of

363 Health Spinal Cord and Brain Injury Research Fund, a Core Facility Grant from the

364 Indiana Clinical and Translational Sciences Institute, Purdue's Office of the Vice 
365 President for Research, and a faculty development grant from Lynchburg College. This

366 material is also based upon work supported by the National Science Foundation

367 Graduate Research Fellowship under Grant No. DGE-1333468. Xenith helmets were

368 provided by Xenith, Inc. We would like to thank the Lynchburg College men's lacrosse

369 program for donating the used helmets tested in the current study. In addition, we thank

370 Jay Hertel, PhD, ATC, FNATA, FACSM for his assistance with the statistical approach

371 to data analysis.

372

373

374

375

376 
Bachynski, K.E., Goldberg, D.S., 2014. Youth sports \& public health: framing risks of mild traumatic brain injury in american football and ice hockey. J. Law. Med. Ethics 42, 323-333.

382 Bartsch, A., Benzel, E., Miele, V., Prakash, V., 2012. Impact test comparisons of 20th and 21st century American football helmets. J Neurosurg 116, 222-233.

384 Bower, D.T., Herbert, E.G., Breedlove, K.M., Pike, A.M., Casa, D.J., Bowman, T.G., In press. Comparing the material properties of various athletic helmet protective outer shells [abstract]. J Athl Train.

387 Bowman, T.G., Breedlove, K.M., Breedlove, E.L., Dodge, T.M., Nauman, E.A., 2015. Force attenuation of new and used lacrosse helmets. J Biomechanics 48, 3782-3787.

Breedlove, E.L., Breedlove, K.M., Bowman, T.G., Nauman, E.A., 2013. Failed Cascade Helmets, in: Oliver, M., NOCSAE (Eds.), Overland Park, KS.

391 Breedlove, E.L., Robinson, M., Talavage, T.M., Morigaki, K.E., Yoruk, U., O'Keefe, K., 392 King, J., Leverenz, L.J., Gilger, J.W., Nauman, E.A., 2012. Biomechanical correlates of symptomatic and asymptomatic neurophysiological impairment in high school football. J. Biomech. 45, 1265-1272.

395 Caswell, S.V., Deivert, R.G., 2002. Lacrosse helmet designs and the effects of impact forces. J Athl Train 37, 164-171.

Dick, R.W., Romani, W.A., Agel, J., Case, J.G., Marshall, S.W., 2007. Descriptive epidemiology of collegiate men's lacrosse injuries: National Collegiate Athletic Association Injury Surveillance System, 1988-1989 through 2003-2004. J Athl Train 42, 255-261.

401 Duma, S.M., Manoogian, S.J., Bussone, W.R., Brolinson, P.G., Goforth, M.W., 402 Donnenwerth, J.J., Greenwald, R.M., Chu, J.J., Crisco, J.J., 2005. Analysis of real-time 403

404 Gadd, C.W., 1966. Use of a weighted-impulse criterion for estimating injury hazard 405 (660793), Proceedings of the Tenth Stapp Car Crash Conference. .

406 Goldsmith, W., Monson, K.L., 2005. The state of head injury biomechanics: past, 407 present, and future part 2: physical experimentation. Crit Rev Biomed Eng 33, 105-207.

408 Guskiewicz, K.M., Mihalik, J.P., Shankar, V., Marshall, S.W., Crowell, D.H., Oliaro, 409 S.M., Ciocca, M.F., Hooker, D.N., 2007. Measurement of head impacts in collegiate 410 football players: relationship between head impact biomechanics and acute clinical 411 outcome after concussion. Neurosurgery 61, 1244-1252; discussion 1252-1243. 
412 Higgins, M.J., Bowman, T.G., 2015. A comparison of head impacts in National 413 Collegiate Athletic Association division 1 and division 3 men's lacrosse athletes 414 [abstract]. J Athl Train 50, S-241 - S-242.

415 Hillman, K., 2005. Introduction to Athletic Training. Human Kinetics, Urbana416 Champaign, IL.

417 Hinton, R.Y., Lincoln, A.E., Almquist, J.L., Douoguih, W.A., Sharma, K.M., 2005. 418 Epidemiology of lacrosse injuries in high school-aged girls and boys; a 3-year 419 prospective study. Am J Sports Med 33, 1305-1314.

420 Hutchinson, T.P., 2014. Peak acceleration during impact with helmet materials: Effects 421 of impactor mass and speed. European journal of sport science 14, S377-S382.

422 Koehling, E.M., Vollavanh, L.R., O’Day, K.M., May, J.M., Bowman, T.G., 2015. Real423 time head impact measurements in intercollegiate men's lacrosse players. [abstract]. $\mathrm{J}$ 424 Athl Train 50, S-242.

425 Levy, M., Ozgur, B., Berry, C., Aryan, H., Apuzzo, M., 2004. Birth and evolution of the 426 football helmet. Neurosurgery 55, 656-662.

427 Lincoln, A.E., Caswell, S.V., Almquist, J.L., Dunn, R.E., Hinton, R.Y., 2013. Video 428 incident analysis of concussions in boys' high school lacrosse. Am J Sports Med 41, $429756-761$.

430 Martin, R.B., Liptai, L., Yerby, S., Williams, K.R., 1994. The relationship between mass 431 and acceleration for impacts on padded surfaces. J. Biomech. 27, 361-364.

432 Martini, D., Eckner, J., Kutcher, J., Broglio, S.P., 2013. Subconcussive head impact 433 biomechanics: comparing differing offensive schemes. Med. Sci. Sports Exerc. 45, 755434761.

435 Mihalik, J.P., Bell, D.R., Marshall, S.W., Guskiewicz, K.M., 2007. Measurement of head 436 impacts in collegiate football players: an investigation of positional and event-type 437 differences. Neurosurgery 61, 1229-1235; discussion 1235.

438 NCAA, 2013. NCAA sports sponsorship and participation rates report: Student-Athlete 439 participation, 1981-1982 - 2012-2013.

440 Newman, J.A., 2015. Design and Testing of Sports Helmets: Biomechanical and 441 Practical Considerations, Accidental Injury. Springer, pp. 755-768.

442 NOCSAE, 2006. Standard linear impactor test method and equipment used in 443 evaluating the performance characteristics of protective headgear and face guards; 444 NOCSAE DOC (ND) 081-04m04.

445 NOCSAE, 2011. Laboratory procedural guide for certifying newly manufactured 446 lacrosse helmets; NOCSAE DOC (ND) 042-04m11a. 
447 NOCSAE, 2012. Standard performance specification for newly manufactured lacrosse 448 helmets with faceguard; NOCSAE DOC (ND) 041-11m12.

449 NOCSAE, 2013a. Standard performance specification for newly manufactured football 450 helmets; NOCSAE DOC (ND) 002-13m13.

451 NOCSAE, 2013b. Standard test method and equipment used in evaluating the 452 performance characteristics of protective headgear/equipment; NOCSAE DOC (ND) 453 001-11m13.

454 O'Day, K.M., Koehling, E.M., Vollavanh, L.R., May, J.M., Bowman, T.G., 2015.

455 Comparison of head impact location during games and practices in intercollegiate men's 456 lacrosse players [abstract]. J Athl Train 50, S-242 - S-243.

457 Ommaya, A.K., Hirsch, A.E., 1971. Tolerances for cerebral concussion from head 458 impact and whiplash in primates. J. Biomech. 4, 13-21.

459 Post, A., Oeur, A., Hoshizaki, B., Gilchrist, M.D., 2013. An examination of American 460 football helmets using brain deformation metrics associated with concussion. Mater. 461 Des. 45, 653-662.

462 Rowson, S., Duma, S.M., 2011. Development of the STAR evaluation system for 463 football helmets: Integrating player head impact exposure and risk of concussion. Ann 464 Biomed Eng 39, 2130-2140.

465 Scher, I., Richards, D., Carhart, M., Thomas, R., Hurlen, N., Lam , T., 2008. Pediatric 466 Head and Neck Injuries in Snow Sports: Evaluating the Influence of Helmets. Journal of 467 ASTM International 5, 1-10.

468 STX, Results of recent NOCSAE $\AA$ standard impact attenuation tests by independent 469 470

471 Talavage, T.M., Nauman, E.A., Breedlove, E.L., Yoruk, U., Dye, A.E., Morigaki, K.E., 472 Feuer, H., Leverenz, L.J., 2014. Functionally-detected cognitive impairment in high 473 school football players without clinically-diagnosed concussion. J. Neurotrauma 31, 327474338 .

475 Vollavanh, L.R., O'Day, K.M., Koehling, E.M., May, J.M., Bowman, T.G., 2015. Effect of 476 impact mechanism on head accelerations in intercollegiate men's lacrosse [abstract]. J 477 Athl Train 50, S-243. 


\begin{tabular}{cccc}
\hline Sport & Brand & Model & Number \\
\hline \multirow{5}{*}{ Football } & $\begin{array}{c}\text { Riddell (Rosemont, } \\
\text { Illinois) }\end{array}$ & $\begin{array}{c}\text { Revolution } \\
\text { Speed }\end{array}$ & 3 \\
& $\begin{array}{c}\text { Schutt (Litchfield, } \\
\text { Illinois) }\end{array}$ & lon 4 & 3 \\
\cline { 2 - 4 } & & \\
\cline { 2 - 4 } & Xenith (Lowell, & X1 & 3 \\
& Massachusetts) & X2E & 3 \\
& & Epic & 3 \\
\hline \multirow{4}{*}{ Lacrosse } & Pro7 & 3 \\
& Cascade (Liverpool, & CPX & 3 \\
& New York) & CPX-R & 3 \\
\cline { 2 - 4 } & & R & 3 \\
\cline { 2 - 4 } & Warrior (Warren, & Trojan & 3 \\
& Michigan) & Venom & 3 \\
\hline
\end{tabular}

Table 1. Helmets included in the study. All helmets were new and never used in athletic activity. 


\begin{tabular}{|l|l|l|l|l|l|l|}
\hline & \multicolumn{3}{|c|}{ Football } & \multicolumn{3}{c|}{ Lacrosse } \\
\hline & $\begin{array}{l}\text { Low } \\
\text { Velocity }\end{array}$ & $\begin{array}{l}\text { Medium } \\
\text { Velocity }\end{array}$ & $\begin{array}{l}\text { High } \\
\text { Velocity }\end{array}$ & $\begin{array}{l}\text { Low } \\
\text { Velocity }\end{array}$ & $\begin{array}{l}\text { Medium } \\
\text { Velocity }\end{array}$ & $\begin{array}{l}\text { High } \\
\text { Velocity }\end{array}$ \\
\hline Front - Front Right Boss & .004 & .058 & .012 & .004 & $<.001$ & $<.001$ \\
\hline Front - Side & .710 & .207 & .012 & $<.001$ & $<.001$ & $<.001$ \\
\hline Front - Right Rear Boss & .191 & .286 & .363 & $<.001$ & $<.001$ & $<.001$ \\
\hline Front - Rear & .873 & .422 & .044 & .293 & .001 & $<.001$ \\
\hline Front - Top & .264 & .018 & .607 & $<.001$ & .004 & .847 \\
\hline Right Front Boss - Side & .288 & .758 & .298 & .966 & .880 & .977 \\
\hline Right Front Boss - Right Rear Boss & .963 & .907 & .377 & .006 & $<.001$ & $<.001$ \\
\hline Right Front Boss - Rear & .266 & .720 & .499 & .336 & .568 & .095 \\
\hline Right Front Boss - Top & .011 & $<.001$ & .003 & $<.001$ & $<.001$ & $<.001$ \\
\hline Side - Right Rear Boss & .003 & .595 & .049 & .003 & .065 & .014 \\
\hline Side - Rear & .531 & .265 & .574 & .422 & .849 & .471 \\
\hline Side - Top & .004 & $<.001$ & $<.001$ & $<.001$ & $<.001$ & $<.001$ \\
\hline Right Rear Boss - Rear & .006 & .764 & .159 & .005 & .014 & .004 \\
\hline Right Rear Boss - Top & $<.001$ & $<.001$ & .075 & $<.001$ & $<.001$ & $<.001$ \\
\hline Rear - Top & .030 & $<.001$ & .003 & $<.001$ & $<.001$ & $<.001$ \\
\hline
\end{tabular}

Table 2. P-values for comparisons of the GSI scores among the different locations during low, medium, and high velocity testing with football and lacrosse helmets. 


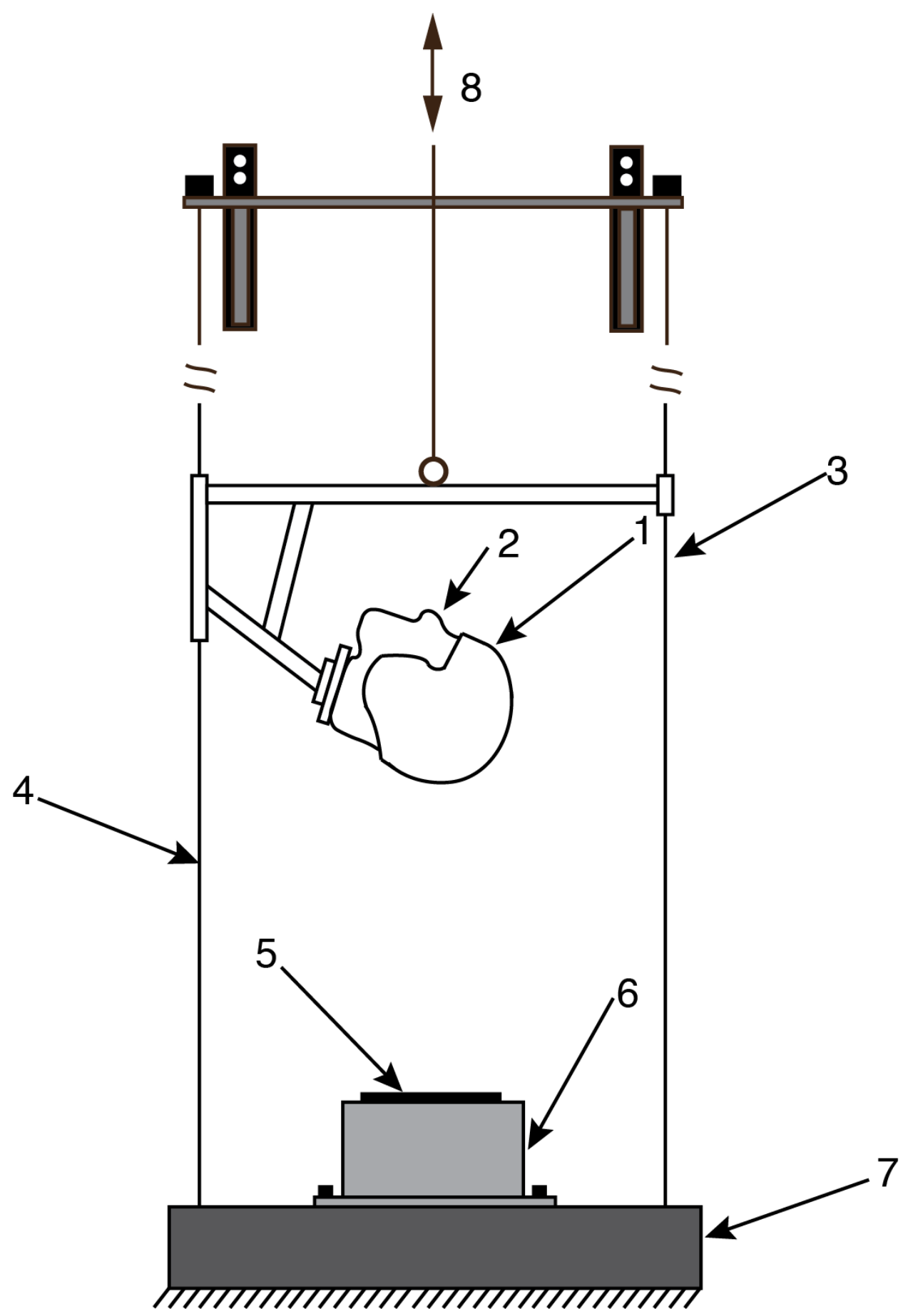

Figure 1. The impact rig consists of a NOCSAE certified head form (1) with the test helmet (2) fitted and secured according to standard protocols. The head form and helmet are attached to the drop carriage frame (3) which slides smoothly over two 1/8" steel cables (7). A motor (8) moves the drop carriage up to the correct height so that it can achieve the pre-set impact velocity following the drop. The head form/helmet strikes a 1/2" MEP test pad secured to an anvil (5) and an anvil base plate (6) for stability. 

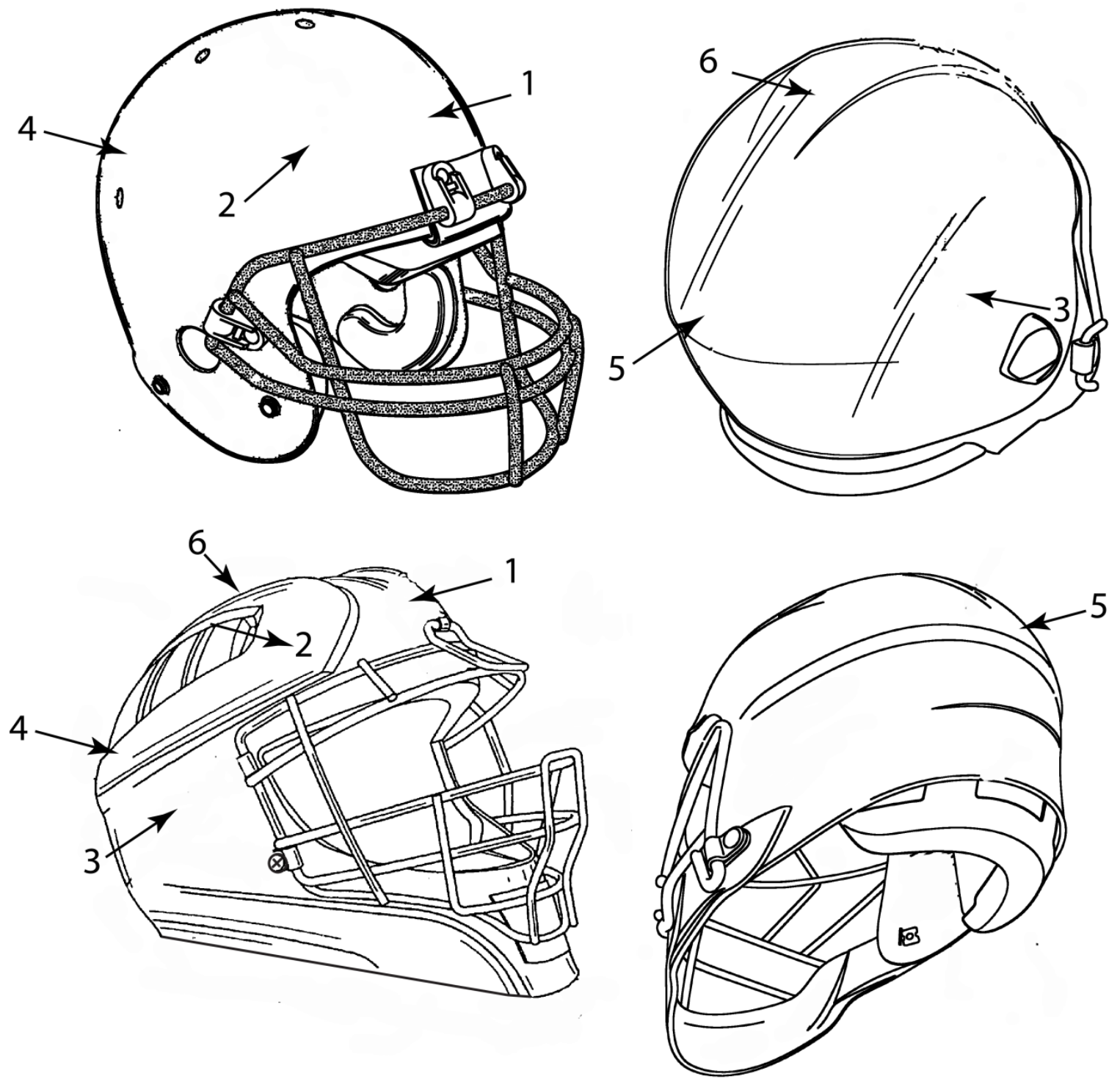

Figure 2. Front and rear views of typical football and lacrosse helmets adapted from US Patent publications 20120291183, 20080163410, 20120297525 , and 20130180034. Each helmet was tested in six locations, (1) front, (2) front boss, (3) side, (4) rear boss, (5) rear, and (6) top as indicated. 

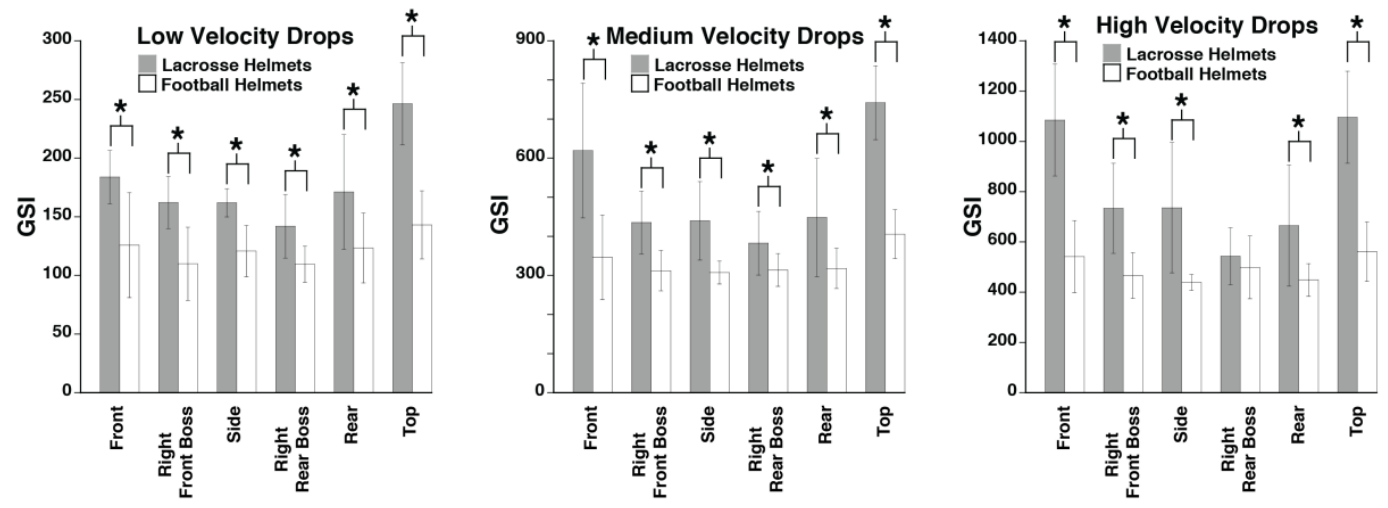

Figure 3. GSI scores of football and lacrosse helmets at the 6 drop locations for low, medium, and high velocity drops. ${ }^{*}$ indicates $p<0.05$. 

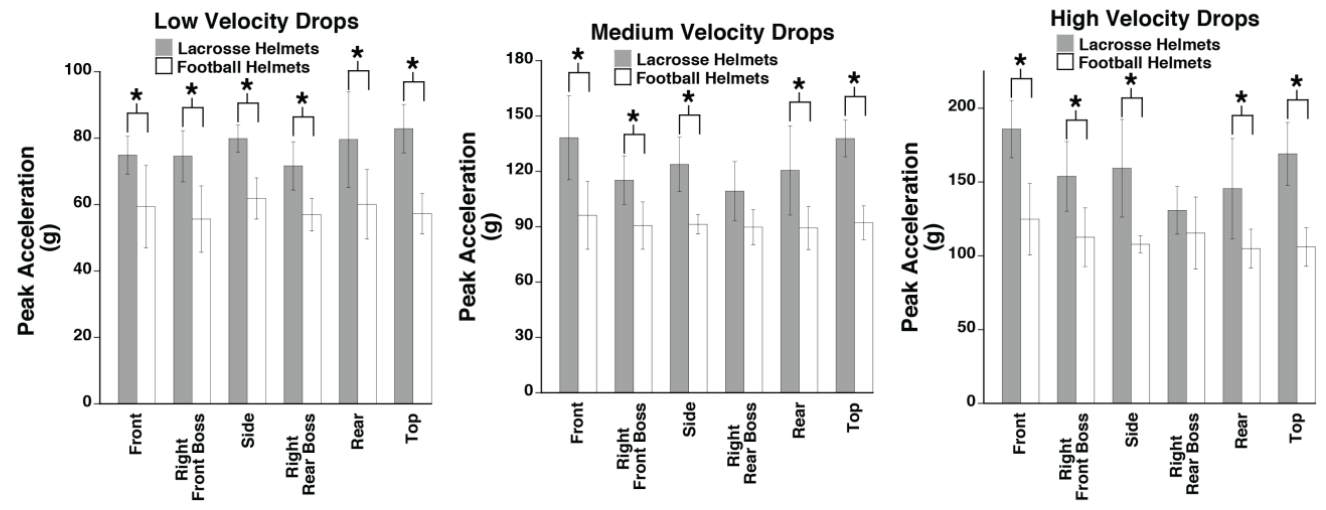

Figure 4. Peak accelerations of football and lacrosse helmets at the 6 drop locations for low, medium, and high velocity drops. ${ }^{*}$ indicates $p<0.05$. 